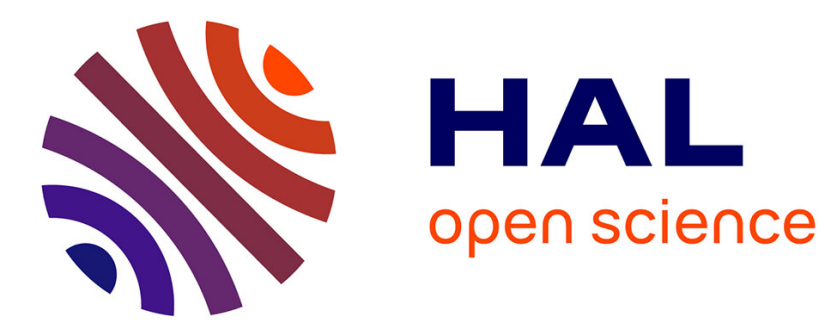

\title{
Multiplexing techniques and applications in fiber-optic spatially resolved sensing networks
}

Daniele Tosi, Carlo Molardi, Wilfried Blanc, Carlos Marques, Salvador Sales

\section{To cite this version:}

Daniele Tosi, Carlo Molardi, Wilfried Blanc, Carlos Marques, Salvador Sales. Multiplexing techniques and applications in fiber-optic spatially resolved sensing networks. OSA Optical Sensors and Sensing Congress, Jun 2019, San Jose, United States. 10.1364/SENSORS.2019.STh4A.2 . hal-02366865

\section{HAL Id: hal-02366865 https://hal.science/hal-02366865}

Submitted on 16 Nov 2019

HAL is a multi-disciplinary open access archive for the deposit and dissemination of scientific research documents, whether they are published or not. The documents may come from teaching and research institutions in France or abroad, or from public or private research centers.
L'archive ouverte pluridisciplinaire HAL, est destinée au dépôt et à la diffusion de documents scientifiques de niveau recherche, publiés ou non, émanant des établissements d'enseignement et de recherche français ou étrangers, des laboratoires publics ou privés. 


\title{
Multiplexing techniques and applications in fiber-optic spatially resolved sensing networks
}

\author{
Daniele Tosi ${ }^{1,2}$, Carlo Molardi $^{1}$, Wilfried Blanc ${ }^{3}$, Carlos Marques $^{4}$, Salvador Sales $^{5}$ \\ ${ }^{I}$ Nazarbayev University, School of Engineering, 010000 Astana, Kazakhstan \\ ${ }^{2}$ National Laboratory Astana, Laboratory of Biosensors and Bioinstruments, 010000 Astana, Kazakhstan \\ ${ }^{3}$ Université Côte d'Azur, INPHYNI-CNRS UMR 7010, Parc Valrose, 06108 Nice, France \\ ${ }^{4}$ Instituto de Telecomunicações, Universidade de Aveiro, Campus Universitário de Santiago, 3810-193, Aveiro, Portugal \\ ${ }^{5}$ Universidad Politecnica de Valencia, iTEAM, Valencia, Spain \\ daniele.tosi@nu.edu.kz
}

\begin{abstract}
Distributed sensing, based on Rayleigh scattering or arrays of reflectors, can be extended as a multi-fiber, multi-sensor technique by using multiplexing features. Current research has overcome the time-and wavelength-division multiplexing, that has represented the golden standard for fiber Bragg gratings. In this work, we introduce novel domain that allow "parallel" multiplexing applied to distributed sensors, and are routed in the use of specialty fibers. The use of fibers doped with $\mathrm{MgO}-$ doped nanoparticles, as well as polymethyl methacrylate fibers, enables new domains for multiplexing, that are discussed in this work. Scattering-level, polarization, and sensitivity based multiplexing are discussed and applied to strain, temperature, and refractive index measurements. OCIS codes: (060.2370) Fiber optic sensors; (290.5870) Scattering, Rayleigh; (060.2310) Fiber optics.
\end{abstract}

\section{Introduction}

Optical fiber sensors (OFS) have been established as a major research trend and as an industry within the last three decades. Historically, the first prototypes of OFS were inherently single-point sensors, capable of measuring one or multiple parameters on a fiber probe. The advent of OTDR (optical time-domain reflectometry) based on Rayleigh, Raman, and Brillouin scattering in fiber provided the first generation of optical fiber sensors, capable of resolving temperature (and, later, strain) over several kilometers of fiber with a resolution that fell from the tens of meters to the current $\sim 10 \mathrm{~cm}$ as data acquisition electronics progressed. Fiber Bragg Grating (FBG) provided a further development, and FBG arrays and sensors are a present industry with significant traction. The significance of FBGs is that, as each grating is a narrow-band reflector, it is possible to stack multiple reflectors within a single fiber and discriminate each grating in the wavelength domain. This approach, labelled time- and wavelength-division multiplexing (TDM, WDM) has allowed gathering hundreds of sensing points in a relatively small area, and therefore has been consolidated into setups that allow fabricating tens of gratings within a single fiber with compact spacing between adjacent sensing elements [1].

In the continuous effort to reduce the spatial resolution of OFS, recent distributed sensing systems have moved away from time-domain detection, and into frequency domain. The OFDR (optical frequency domain reflectometry) principle has introduced substantial innovations, as this method is capable of interrogating the multiple reflections occurring in an optical fiber cable, with resolution ranging between $<1 \mathrm{~mm}$ to $1 \mathrm{~cm}$ for most cases. OFDR can be applied to incoherent systems, which have spatial resolution limited by the coherency length of the optical source, and to coherent systems in which the spatial resolution is limited by the window size of the processing unit.

An important case of coherent OFDR is the so-called OBR (optical backscatter reflectometer), which is capable of resolving the Rayleigh scattering that occurs in the fiber [2]. This system allows working with standard single mode fibers as sensors, rather than with cascade of reflectors such as FBG arrays, all-grating fibers, or microstructured fibers, thus minimizing the complexity of the sensor. The OBR detects the spectrum of the Rayleigh scattering in each point of the fiber, which appears as a random signal (known as "signature") that shifts when temperature or strain variations are applied to the fiber. Such system, capable of working at the millimeter-scale resolution, has attracted the interest for biomedical applications, such as strain sensing in percutaneous and endoscopic catheters, and monitoring minimally invasive thermo-therapies.

Sensing features could be significantly extended, if we were able to alter the OBR architecture to a multi-fiber, multiplexed system; as a matter of fact, the OBR is a single-channel instrument. However, by using specialty fibers we can make significant steps forward in terms of sensing potential, and introduce new mechanism for multiplexing that can operate with signals that are inherently wideband such as Rayleigh scattering. 

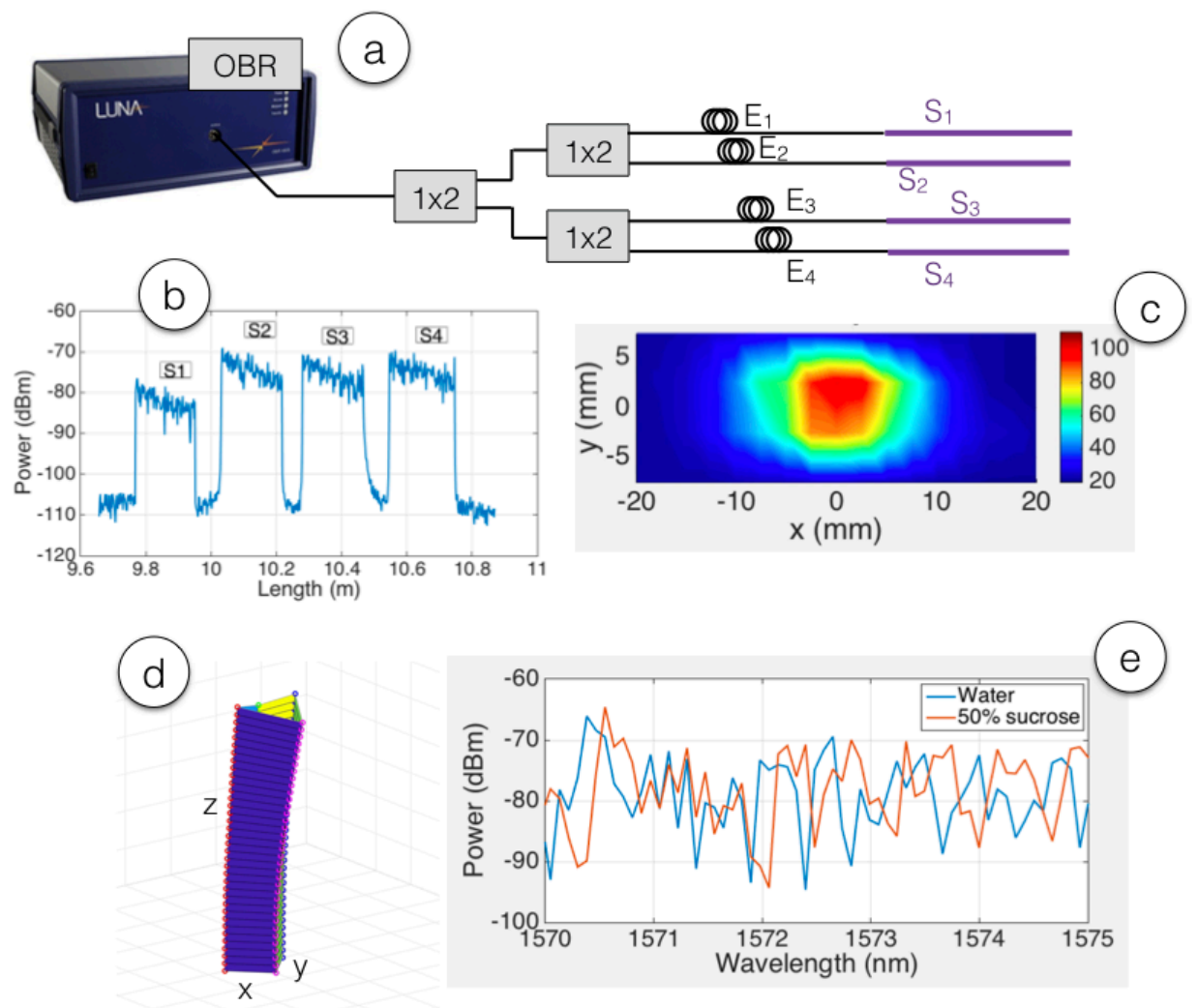

Fig. 1. Overview of the distributed sensing multiplexing concepts. (a) Implementation of the scattering-level multiplexing, arranged with fiber extenders $\left(\mathrm{E}_{1} \ldots \mathrm{E}_{4}\right)$ and sensing fibers $\left(\mathrm{S}_{1} \ldots \mathrm{S}_{4}\right)$. (b) Scattering trace of the fiber parallel array, with $\mathrm{MgO}$-doped fibers. (c) $2 \mathrm{D}$ temperature rendering with an array of 4 fibers. (d) 3D shape sensing of an epidural needle performed with 4 sensing fibers mounted on the cardinal points of the needle. (e) Refractive index sensitivity of the scattering traces after etching the sensing fibers.

In Fig. 1, we show some of the recent results to be discussed. A first significant achievement is obtained by using fibers having $\mathrm{MgO}$-doped nanoparticles within the fiber core [3]. The enhancement of Rayleigh scattering in this fiber allows the parallel multiplexing arrangement described in Fig. 1(a). From this setup, we can interrogate multiple fibers with a simultaneous scan and obtain strain or temperature patterns on a plurality of fibers. Knowing the position of the sensing fibers, and that we can discriminate each strain or temperature contribution in a 2- or 3-dimensional coordinate system, we can perform an advanced sensing of temperature, strain, shape, or even refractive index by etching the sensing fiber. In the presentation, we also discuss the influence of polymer fibers operating in the infrared, which yield a higher sensitivity and allow a further discrimination of temperature and strain.

\section{Conclusions}

In conclusion, this work presents techniques for parallel multiplexing using specialty fibers, which customize scattering properties and sensitivity features. Applications in biomedical engineering are also discussed, such as temperature 2D/3D rendering, strain and shape sensing, and refractive index sensing.

\section{References}

[1] D. Tosi et al., "Fiber optic sensors for sub-centimeter spatially resolved measurements: review and biomedical applications," Opt. Fiber Technol. 43, 6-19 (2018).

[2] M. Froggatt and J. Moore, "High-spatial-resolution distributed strain measurement in optical fiber with Rayleigh scatter," Appl.Opt. 37, 1735 (1998).

[3] W. Blanc et al., "Fabrication of Rare Earth-Doped Transparent Glass Ceramic Optical Fibers by Modified Chemical Vapor Deposition," $J$. Am. Ceram. Soc. 94, 2315-2318.(2011) 